\title{
Encore sur la « marelle ronde » : cent ans après Carl Blümlein
}

Ulrich Schädler

\section{(2) OpenEdition}

1 Journals

Édition électronique

URL : http://journals.openedition.org/kentron/2666

DOI : 10.4000/kentron.2666

ISSN : 2264-1459

Éditeur

Presses universitaires de Caen

\section{Édition imprimée}

Date de publication : 18 décembre 2018

Pagination : 87-98

ISBN : 978-2-84133-902-0

ISSN : 0765-0590

\section{Référence électronique}

Ulrich Schädler, « Encore sur la « marelle ronde » : cent ans après Carl Blümlein », Kentron [En ligne], 34 | 2018, mis en ligne le 20 décembre 2018, consulté le 18 novembre 2020. URL : http:// journals.openedition.org/kentron/2666 ; DOI : https://doi.org/10.4000/kentron.2666

\section{(c) (i) (9)}

Kentron is licensed under a Creative Commons Attribution-NonCommercial-NoDerivatives 3.0 International License. 


\section{ENCORE SUR LA "MARELLE RONDE»: CENT ANS APRÈS CARL BLÜMLEIN ${ }^{1}$}

Parmi les nombreux motifs gravés dans les pavés marbrés des villes romaines, le cercle traversé de quatre lignes qui se coupent en son centre est certainement le motif le plus fréquent ${ }^{2}$. Pendant longtemps, on ne savait pas comment interpréter ces «roues à rayons». Il y a un peu plus d'un siècle, l'idée est née qu'il s'agit là d'une version ronde de la petite marelle (ou mérelle) à trois pions («three men's morris»). Ce jeu, paraît-il, a été décrit brièvement par Ovide, qui en parle dans Ars amatoria III, 365-366, et Tristia, II, 480-481. Dans son Libro de los juegos de 1284, Alphonse $\mathrm{X}$ en donne une description plus détaillée ainsi qu'une illustration au fol. $95 \mathrm{a}^{3}$. Cependant, la petite marelle se joue sur un plateau carré.

L'idée de la petite marelle ronde est née autour de 1900. Par exemple, pour Émile Boeswillwald, une "roue» inscrite dans les dalles du forum de Timgad était sans doute un «jeu de marelle» (fig. 1, page suivante): «Pour terminer, nous signalerons, gravé sur le dallage en $m$, un jeu de marelle, dont nous donnons le dessin ci-contre. Le diamètre du cercle est à peu près de o m.6o. On sait que le sol de la basilique julienne, sur le forum romain, offre de nombreux exemples de figures semblables. Il fallait bien, à Timgad comme à Rome, que les désœuvrés s'occupassent; dans les provinces aussi bien que dans la capitale il y avait des gens "assez pervers, comme disait Cicéron, pour oser jouer aux dés même au forum" " ${ }^{4}$.

Toutefois, encore en 1913, Harold J.R. Murray ne voyait pas quelle interprétation on pouvait donner à ces dessins: il plaçait le modèle circulaire à côté de la version carrée avec la légende "Boards for the Smaller Merels», mais avouait que

1. Cet article est issu des recherches soutenues par le projet ERC (European Research Council) Locus Ludi. The Cultural Fabric of Play and Games in Classical Antiquity, implanté à l'université de Fribourg, http://locusludi.unifr.ch.

2. Voir Schädler 2012, 21-22.

3. Schädler \& Calvo 2009, 295-296; 298-299.

4. Boeswillwald et al. 1905, 27. 


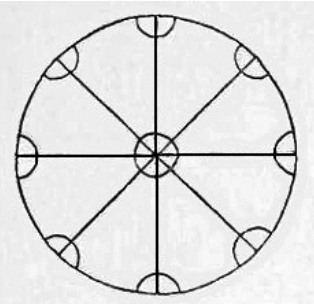

Fig. 1 - «Jeu de marelle» à Timgad,

d'après Boeswillwald et al. 1905, p. 27

«their purpose is unknown ${ }^{5}$. C'est Carl Blümlein qui donne finalement les règles de ce jeu et un exemple de partie (fig. 2), inspiré de Becq de Fouquières, qui l'avait proposé pour la version carrée - une interprétation qui a tout de suite convaincu (fig. 3$)^{6}$.

Ainsi, Hans Lamer écrivait en 1927 que la petite marelle ronde était le seul jeu de pions antique dont les règles étaient conservées ${ }^{7}$. Aujourd'hui, la petite marelle ronde est souvent considérée comme le jeu de pions le plus populaire des Romains; les médiateurs et médiatrices culturelles des musées archéologiques y jouent avec leurs jeunes visiteurs, et de nombreuses éditions commerciales sont mises en vente.

En 2007, dans une conférence intitulée "Ashtapada and round smaller merels: two newly invented "ancient" board games?», donnée à l'occasion du $\mathrm{X}^{\mathrm{e}}$ colloque «Board Game Studies» à Sankt Pölten (Autriche), j’ai exprimé mes doutes concernant l'interprétation des «wheel patterns» comme plateaux de jeu pour une version ronde de la petite marelle à trois pions. J'ai relevé que ce jeu n'est attesté nulle part dans le monde et que des « roues» d'entre 70 et $120 \mathrm{~cm}$ de diamètre gravées dans les dalles de la Basilica Iulia ne permettent certainement pas de placer trois petits pions en ligne. J'avançais l'hypothèse que ce jeu était plutôt une invention des archéologues modernes.

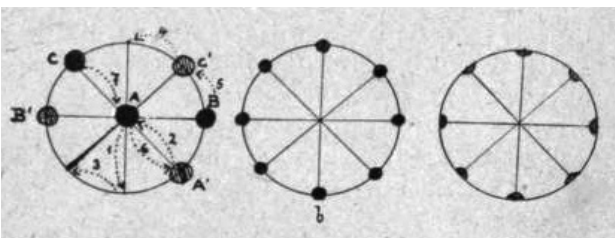

Fig. 2 - Exemple de partie de la «marelle ronde», d'après Blümlein 1918, p. 101

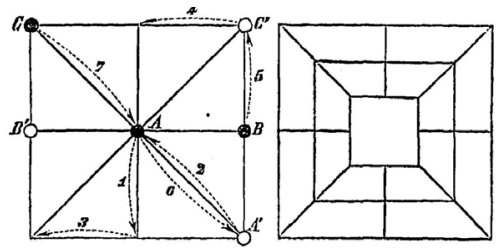

Fig. 3 - Exemple de partie de la petite marelle, d'après Becq de Fouquières 1869, p. 390

5. Murray 1913, 614; à comparer avec Murray 1952, 38-42, mais voir 18, note 3, avec fig. 7 B.

6. Blümlein 1918, 101. À comparer avec Becq de Fouquières 1869, 389-390.

7. Lamer 1927. 


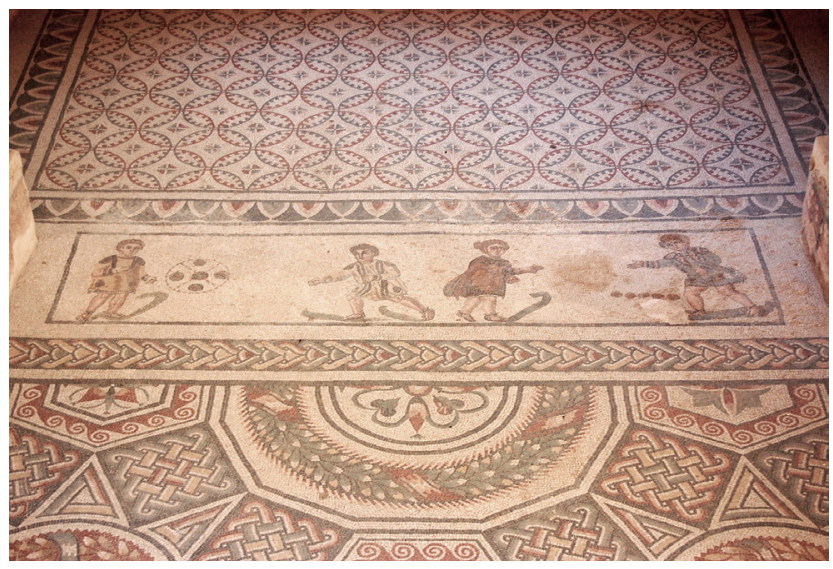

Fig. 4 - Jeu de lancer, Villa del Casale,

Piazza Armerina (photo U. Schädler)

J'étais conforté dans mon opinion par une étude que Florian Heimann avait soumise au Board Game Studies Journal en 2005, mais publiée, à cause de l'arrêt de la publication de la revue au format papier, seulement en 2014 dans sa version en ligne $^{8}$. Il a montré que la règle proposée par Carl Blümlein en 1918 avait de graves défauts, qui ont pour conséquence que le jeu, en réalité, ne fonctionne pas.

Indépendamment de Heimann, Claudia-Maria Behling est arrivée aux mêmes conclusions et a suggéré que les "roues» auraient servi de plateaux pour un jeu similaire au «franc du carreau » ${ }^{9}$. Ce jeu, mentionné entre autres par Rabelais au $\mathrm{XVI}^{\mathrm{e}}$ siècle et illustré par Stella un siècle plus tard ${ }^{10}$, consiste effectivement à lancer des pièces de monnaie ou des palets sur un plateau identique à la petite marelle: un carré divisé par quatre lignes verticales, horizontales et diagonales. Le but du jeu est de les lancer avec l'intention de trouver le centre ou de placer la pièce le plus proche du centre sans qu'elle touche une ligne (ou bien, comme l'explique le Dictionnaire de Trévoux, «sur l'endroit le plus éloigné des raies ou bords du carreau» ${ }^{11}$ ). Le terme «franc» ne fait bien sûr pas référence au type de monnaie; c'était ce placement dégagé à l'intérieur du carré que l'on appelait «franc».

Cette proposition de Behling est effectivement confirmée par la représentation de ce jeu sur une des mosaïques de la Villa del Casale à Piazza Armerina, une représentation qui lui a échappé (fig. 4).

\footnotetext{
8. Heimann 2014.

9. Behling 2013; Behling 2014, part. 65.

10. Esmangart \& Johanneau 1823, 406 avec note 56; Bouzonnet Stella \& Stella, 1657, 17.

11. Brillant 1771, 290.
} 


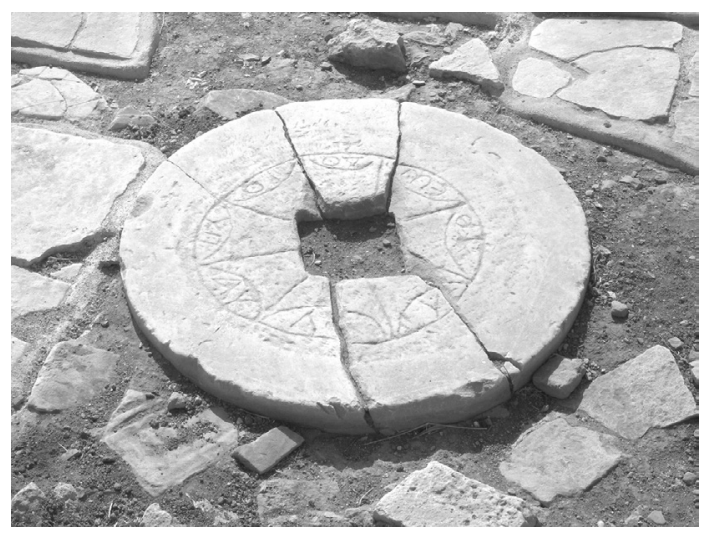

Fig. 5 - Topos marker de Paulos Theodoros, Samos, sanctuaire de Héra (photo U. Schädler)

Sur la mosaïque, sont représentés deux jeux différents. À droite, un garçon lance des palets pour qu'ils forment une ligne droite. À gauche, en revanche, un autre garçon lance des palets dans un cercle divisé par deux lignes, dessiné par terre. Les quatre sections créées par les lignes à l'intérieur du cercle sont déjà occupées par quatre palets, qui ne touchent pas les lignes et donc se trouvent exactement dans une position «franche». Le garçon vient de lancer le cinquième palet au centre du plateau, là où les lignes se croisent. La position des palets correspond parfaitement aux descriptions et règles $\mathrm{du}$ " franc du carreau », dont on identifie ici une version ronde. Il est évident qu'une variante plus difficile consisterait à jouer avec un plateau traversé par huit rayons au lieu de quatre.

Cependant, les «roues» sont effectivement trop nombreuses pour avoir servi exclusivement comme plateau pour le «franc du carreau ». D'autres fonctions sont fort probables; j'en évoque ici quelques-unes, sans vouloir traiter cette thématique de façon exhaustive. Ainsi, Charlotte Roueché a proposé de voir dans certains de ces motifs des «topos markers ${ }^{12}$. Il s'agirait de symboles gravés dans le pavement pour indiquer la place destinée à une personne, comme, par exemple, à un marchand, ou à des participants à des cérémonies ou à d'autres manifestations. Deux exemples portant des inscriptions confirment cette interprétation: sur le marché de Philippes, on trouve une «roue» avec l'inscription I $\Omega$ ANNOY МАГPOY, c'est-à-dire «(la place de) Jean le boucher ${ }^{13}$. Et, dans le sanctuaire de Héra de Samos, se trouve une «roue» estampillée ПA $\Lambda$ OY $\Theta E \Omega \Delta O P O Y$, c'est-à-dire «(la place de) Paulos Theodoros» (fig. 5).

12. Roueché \& Chaisemartin 1993.

13. Coupry 1946. 


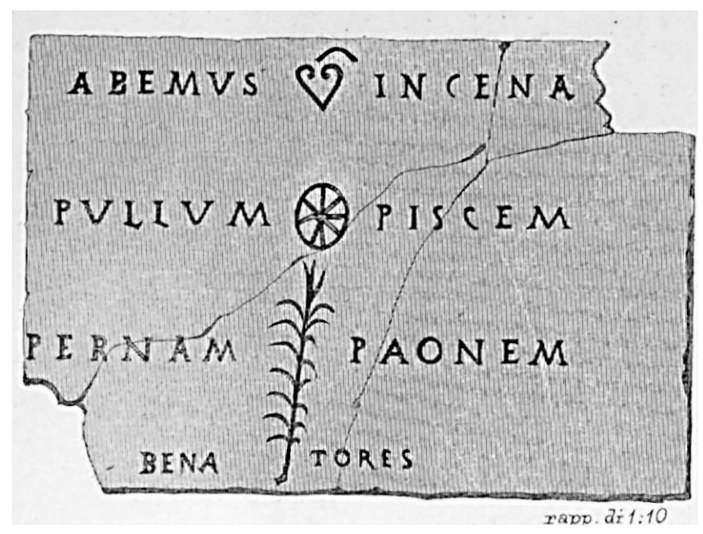

Fig. 6 - Plaque en marbre, plateau du Ludus duodecim scriptorum?

Musei Capitolini, d'après Lanciani 1876, Tav. XXI, fig. 1

Le motif de la roue apparaît aussi dans des contextes liés à une symbolique de bonheur ou de victoire. Sur la fameuse plaque de marbre - s'agit-il d'un véritable plateau de jeu ou bien n'est-ce qu'un panneau utilisé comme plateau de jeu? - servant pour le jeu des XII Scripta/Alea des venatores ${ }^{14}$, conservée aux musées du Capitole et portant l'hexagramme "ABEMUS IN CENA / PULLUM PISCEM / PERNAM PAONEM», les symboles séparant les couples de mots au milieu sont les suivants: un rameau de palmier, une feuille et une «roue» $(\text { fig. } 6)^{15}$. Le palmier, symbole par excellence de la victoire, est offert aux auriges vainqueurs des courses au cirque. Les feuilles représentent une forme abrégée de la couronne de laurier et se trouvent dans le contexte de représentations de gladiateurs victorieux. Parmi les graffitis trouvés en 1904 sous la Via Nazionale à Rome, deux gladiateurs lèvent une feuille avec la main droite ${ }^{16}$. Les deux mosaïques des gladiateurs provenant de la Voie Appienne et conservées au Musée archéologique national de Madrid en donnent un autre exemple: la phrase "Astyanax vicit» sur MAN 3600 ainsi que l'acclamation «Symmachi homo felix» sur MAN 3601 sont accentuées par une feuille ${ }^{17}$. Avec le rameau de palmier, les feuilles de laurier forment le couple "palma et laurus ${ }^{18}$. Il est donc fort probable que, sur le plateau des venatores, la «roue» serve également de symbole de victoire et de bonheur.

14. La plaque est «signée» par les «benatores»: «venatores».

15. Lanciani 1876, Tav. XXI, fig. 1. Voir la description de cette figure, 188-189.

16. Gatti 1904, fig. 2 et 4 .

17. Blake 1940, 112-113; Oliver 1957, 13; Fagan 2011, 138. Les inscriptions: CIL VI $10205=$ VI $33979=$ ILS $5140=$ EAOR 1.114.

18. Marrou 1941; Coche de la Ferté 1961, 139. 


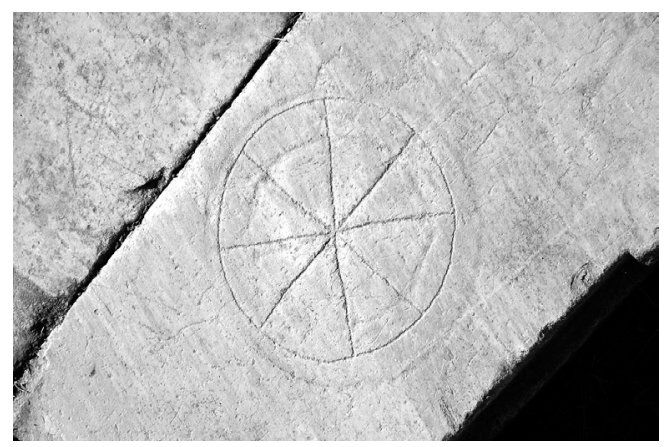

Fig. 7 - «Roue à rayons», Éphèse, Arkadiané (photo U. Schädler)

Apparemment, cette même signification est indiquée par l'inscription "NIKA $\mathrm{H}$ TYXH O $\Lambda \Upsilon \mathrm{M}[\ldots] \Delta \mathrm{O} \Sigma$ » autour d'une « roue» se trouvant à Éphèse, dans la rue qui mène du port au théâtre, appelée l'Arkadiané ${ }^{19}$. Sur l'Embolos, autre grande voie d'Éphèse, à l'est de la fontaine de Trajan et sur le côté nord de la rue, se trouvent une petite « roue» et, juste à côté, l'acclamation «NIKA H TYXH ПPA $\Sigma I N \Omega N$ » en faveur de la faction des verts ${ }^{20}$. Une autre, de nouveau dans l'Arkadiané, porte la prière $\mathrm{K} Y \mathrm{PIE} \mathrm{BOH} \Theta \mathrm{E} \Sigma \mathrm{ON}^{21}$. Le motif de la roue accompagne donc des acclamations et des prières et place même les affaires de Jean et Paul ${ }^{22}$ sous de bons auspices. Outre cette symbolique positive, les roues pouvaient également, semble-t-il, assumer une fonction apotropaïque: en effet, on les trouve souvent placées directement devant des entrées ou bien gravées dans des seuils. Peut-être que l'on peut associer aussi un motif de roue sur les gradins de la Basilica Iulia à Rome. Un cercle, dont les huit rayons ne sont indiqués que par des traits courts, entoure l'inscription «Senatus populus(que) [Romanus ex] oraculo» (CIL VI 29850) ${ }^{23}$. Ce graffito, tracé de manière improvisée par un inconnu, reprend le texte d'un groupe d'inscriptions bilingues trouvé à proximité et se référant également à un oracle (IGUR 94-97).

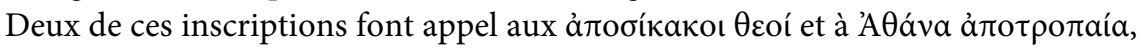
et sont donc clairement liées à une crise ou à un désastre, peut-être la peste de 166.

19. Schädler 2012, 22, fig. 7 .

20. Engelmann et al. 1980, n ${ }^{\circ} 1198$. Rappelons que la faction des verts désigne l'écurie des verts dans le cirque, pour les courses de chars; les autres factions étant celles des blancs, des rouges et des bleus.

21. Lessing \& Oberleitner 1978, fig. 62.

22. Jean et Paul étant les deux personnages mentionnés dans les «topos markers» décrits plus haut: Johannes le boucher à Philippes, et Paulos Theodoros à Samos (cf. p. 4).

23. Huelsen 1894, 91-92; Aronen 1983; Kajava 2007. 


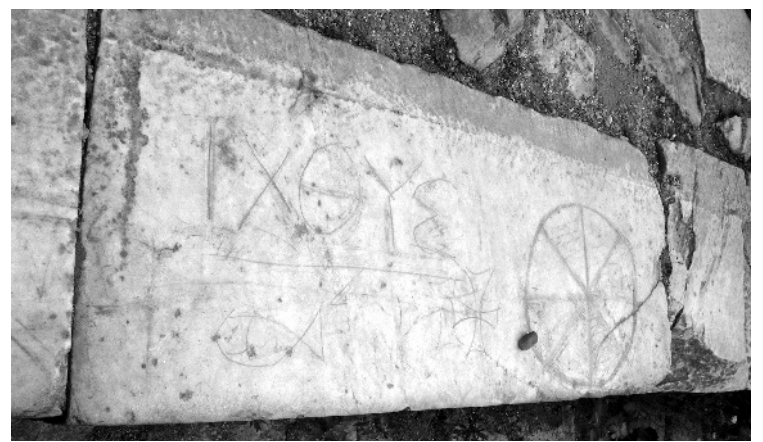

Fig. 8 - «Roue à rayons» interprétée par des guides touristiques, Éphèse, Staatsmarkt (Wikimédia Commons)

Le motif de la roue entourant ce graffito semble avoir eu la fonction de renforcer l'effet des mesures prises à la suite de l'oracle et confirmerait sa signification apotropaïque et sotériologique. Cet exemple ne peut donc pas être utilisé pour soutenir l'hypothèse d'une fonction des "roues" comme supports d' "oracles informels", proposée par Olaf Höckmann pour les motifs gravés dans le stylobate du temple d'Apollon à Didyme ${ }^{24}$. Même s'il est difficile de prouver cette hypothèse sans indications complémentaires, certaines procédures ou rituels semblent avoir été exécutés autour de ces dessins. À Éphèse, on observe, sur l'Arkadiané, le cas d'un motif de roue gravé avec un outil pointu, comme c'est le cas normalement. Mais autour et à l'intérieur de cette "roue» se trouvent deux autres cercles tracés avec l'aide d'un objet plutôt émoussé, probablement déplacé maintes fois pour produire ce creux dans le marbre (fig. 7). De plus, la concentration de plusieurs de ces «roues» sur une surface limitée, que l'on trouve souvent dans les villes d'Éphèse, d'Aphrodisias et d'Éleusis ${ }^{25}$ entre autres, laisse à penser qu'il était important de ne pas seulement «utiliser», le moment donné, une «roue» déjà existante, mais de produire un tel motif de nouveau, si besoin était.

On ne peut pas non plus exclure une symbolique chrétienne pour certains de ces motifs. Les guides touristiques à Éphèse expliquent aux visiteurs qu'il s'agit d'un monogramme symbolisant IX $\Theta Y C$ ou IX $\Theta \Upsilon \Sigma$ (fig. 8), obtenu par la superposition des lettres. Cette idée semble étayée par une grande plaque ronde en alabastre trouvée dans la Villa dei Quintili sur la Voie Appienne et exposée dans le musée du site (inv. 67693) ${ }^{26}$ : une «roue» à huit rayons et, en dessous, la légende IX $\Theta Y C$ dans une tabula ansata sont soigneusement gravées dans la plaque.

24. Höckmann 1996, en part. 257-258; Widura 2015, 73.

25. Roueché 2007; Widura 2015, 77-81, Abb. 17.

26. Schädler 1998, $114 \mathrm{n}^{\circ} 104$. 


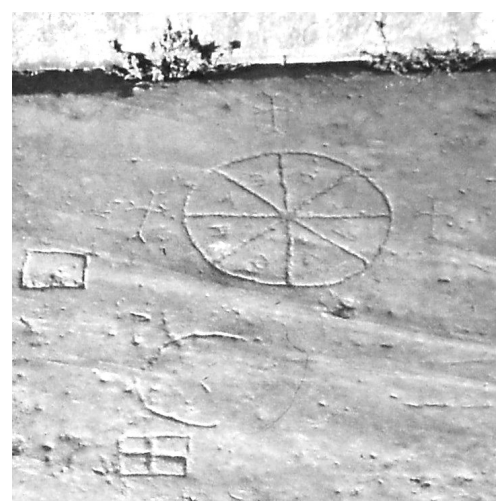

Fig. 9 - « Roue à rayons» et croix chrétiennes, Didyme, temple d'Apollon (photo U. Schädler)

Une autre piste serait indiquée par des dédicaces à Hélios trouvées à Épidaure portant comme symbole du dieu soleil un cercle avec huit rayons - tout à fait similaire à une "roue» ${ }^{27}$. Même si le chrisme consiste normalement en un cercle divisé par des lettres $\mathrm{X}$ et Rho superposées, qui font donc six rayons au lieu de huit, on peut imaginer que par l'association Hélios-Christus ${ }^{28}$ les «roues» pourraient avoir eu une signification chrétienne. À Didyme, par exemple, une «roue» avec, dans les tranches, des lettres difficilement déchiffrables, est entourée de trois croix chrétiennes (fig. 9).

En conclusion, les nombreuses « roues " gravées dans les pavements de marbre des villes antiques avaient certainement des fonctions et valeurs symboliques différentes. Parmi ces fonctions, celle d'un plateau de jeu pour un jeu de lancer similaire au «franc du carreau » est très vraisemblable, car attestée par l'iconographie. Cent ans après Blümlein, l'hypothèse d'une «marelle ronde» devrait donc être définitivement écartée.

Ulrich SCHÄDLER Musée suisse du Jeu, La Tour-de-Peilz ERC Locus Ludi. The Cultural Fabric of Play and Games in Classical Antiquity Université de Fribourg

27. Blinkenberg 1899, p. $386 \mathrm{n}^{\text {os }} 152$ et 258 .

28. Voir, par exemple, Knipp 1998, 41 sq.; Michel 2004, 118 Anm. 626. 


\section{Références bibliographiques}

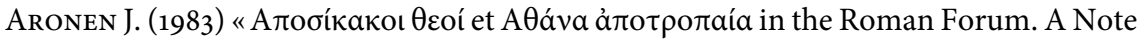
on IGUR 94-95», Arctos, $\mathrm{n}^{\circ}$ 17, p. 5-11.

BeCQ De Fouquières L. (1869), Les jeux des Anciens. Leur description, leur origine, leurs rapports avec la religion, l'histoire, les arts et les mours, Paris, C. Reinwald.

Behling C.-M. (2013), «Le mystère de la marelle ronde», Archéothéma, n 31, Jeux et jouets gréco-romains, p. 47.

Behling C.-M. (2014), «Der sog. Rundmühle auf der Spur - Zug um Zug zur Neudeutung römischer Radmuster», in Akten des 14. Österreichischen Archäologentages am Institut für Archäologie der Universität Graz vom 19. bis 21. April 2012, E. Trinkl (dir.), Vienne, Phoibos Verlag, p. 63-70.

Blake M.E. (1940), «Mosaics of the Late Empire in Rome and Vicinity», Memoirs of the American Academy in Rome, vol. 17, p. 81-130.

Blinkenberg C. (1899), «Epidaurische Weihgeschenke IV», MDAI(A), vol. 24, p. 379-397.

BLÜMLEIN C. (1918), Bilder aus dem römisch-germanischen Kulturleben, Munich, Oldenburg.

Boeswillwald É., Cagnat R., Ballu A. (1905), Timgad. Une cité africaine sous l'Empire Romain, Paris, Leroux.

Bouzonnet Stella C., Stella J. (1657), Les jeux et plaisirs de l'enfance, Paris, Stella.

BRILlANT abbé (éd.) (1771), Dictionnaire universel françois et latin, vulgairement appelé Dictionnaire de Trévoux [1704], vol. 4, Paris, Compagnie des libraires associés.

Coche de la Ferté É. (1961), «Palma et Laurus. Un monogramme et un objet d'art paléochrétiens en relation avec les courses de l'hippodrome», Jahrbuch der Berliner Museen, vol. 3, p. 134-147.

Coupry J. (1946), «Un joueur de marelle au marché de Philippes», BCH, vol. 70, p. 102-105.

Engelmann H., Knibbe D., Merkelbach R. (1980), Die Inschriften von Ephesos, T. 4, Bonn, Habelt (Inschriften griechischer Städte aus Kleinasien; 14).

Esmangart C., Johanneau É. (éd.) (1823), Euvres de Rabelais, t. 1, Paris, Dalibon.

FAgAn G.G. (2011), The Lure of the Arena. Social Psychology and the Crowd at the Roman Games, Cambridge - New York, Cambridge University Press.

GAтTi G. (1904), «Nuove scoperte nella città e nel suburbio », NSA, p. 153-157.

Heimann F. (2014), «The Loop within Circular Three Men’s Morris» (introd. U. Schädler), Board Game Studies Journal Online, n 8 , p. 51-61.

Höckmann O. (1996), «Brettspiele im Didymeion», MDAI(I), vol. 46, p. 251-262. 
Huelsen C. (1894), «Ein Graffito vom Forum Romanum», $M D A I(R)$, vol. 9, p. 91-93.

Kajava M. (2007), "Ex oraculo", in Res bene gestae. Ricerche di storia urbana su Roma antica in onore di Eva Margareta Steinby, A. Leone, D. Palombi, S. Walker (dir.), Rome, Edizioni Quasar (Lexicon Topographicum Urbis Romae, Supplementum; 4), p. 127-132.

KNIPP D. (1998), Christus Medicus in der frühchristlichen Sarkophagskulptur. Ikonographische Studien zur Sepulkralkunst des späten vierten Jahrhunderts, Leyde, Brill.

LAMER H. (1927), «Lusoria tabula», RE, XIII/2, col. 1900-2029.

Lanciani R. (1876), "Ara di Vermino», Bullettino della Commissione archeologica comunale di Roma, $\mathrm{n}^{\circ}$ 4, p. 165-210.

Lessing E., Oberleitner W. (1978), Ephesos. Weltstadt der Antike, Vienne - Heidelberg, Veberreuter.

Marrou H.I. (1941), " "Palma et Laurus” », MEFR, t. 58, p. 109-131.

Michel S. (2004), Die Magischen Gemmen. Zu Bildern und Zauberformeln aufgeschnittenen Steinen der Antike und Neuzeit, Berlin, Akademie-Verlag (Studien aus dem Warburg-Haus; 7).

Murray H.J.R. (1913), A History of Chess, Oxford, Oxford University Press.

Murray H.J.R. (1952), A History of Board Games other than Chess, Oxford, Clarendon Press.

Oliver J.H. (1957), "Symmachi, Homo Felix», Memoirs of the American Academy in Rome, vol. 25, p. 7-15.

Roueché C. (2007), "Late Roman and Byzantine Game Boards at Aphrodisias », in Ancient Board Games in Perspective. Papers from the 1990 British Museum Colloquium, with Additional Contributions, I.L. Finkel (éd.), Londres, British Museum Press, p. 100-105.

Roueché C., Chaisemartin N. de (1993), Performers and Partisans at Aphrodisias in the Roman and Late Roman Periods, Londres, Society for the Promotion of Roman Studies (Journal of Roman Studies Monograph; 6).

SCHÄDlER U. (1998), "Scavi e scoperte nella Villa dei Quintili», in La Villa dei Quintili. Fonti scritte e fonti figurate, A. Ricci (dir.), Rome, Lithos, p. 29-234.

SChädler U. (2012), "Jouer par terre», in Art du jeu, jeu dans l'art. De Babylone à l'Occident médiéval, Catalogue de l'exposition au Musée de Cluny, I. Bardiès-Fronty, A.-E. Dunn-Vaturi (dir.), Paris, Réunion des musées nationaux - Grand Palais, p. 20-23. 
ENCORE SUR LA «MARELlE RONDE»: CENT ANS APRÈs CARL BLÜMLEIN

Schädler U., Calvo R. (éd.) (2009), Alfons X. «der Weise». Das Buch der Spiele, Vienne - Berlin, LIT (Ludographie - Spiel und Spiele; 1 ).

Widura A. (2015), SpielRäume. Kulturhistorische Studien zum Brettspiel in archäologischen Kontexten, Rahden, Marie Leidorf. 